\title{
Effects of Balance Training on Balance Performance in Healthy Older Adults: A Systematic Review and Meta-analysis
}

\author{
Melanie Lesinski ${ }^{1}$ Tibor Hortobágyi ${ }^{2}$. Thomas Muehlbauer ${ }^{1} \cdot$ Albert Gollhofer $^{3}$. \\ Urs Granacher ${ }^{1}$
}

Published online: 1 September 2015

(C) The Author(s) 2015. This article is published with open access at Springerlink.com

\begin{abstract}
Background The effects of balance training (BT) in older adults on proxies of postural control and mobility are well documented in the literature. However, evidence-based dose-response relationships in BT modalities (i.e., training period, training frequency, training volume) have not yet been established in healthy older adults.

Objectives The objectives of this systematic literature review and meta-analysis are to quantify BT intervention effects and to additionally characterize dose-response relationships of BT modalities (e.g., training period, training frequency) through the analysis of randomized controlled trials (RCTs) that could maximize improvements in balance performance in healthy communitydwelling older adults.
\end{abstract}

This article is part of the Topical Collection on Exercise to improve mobility in healthy aging.

Melanie Lesinski

mlesinsk@uni-potsdam.de

1 Division of Training and Movement Sciences, Research Focus Cognition Sciences, University of Potsdam, Am Neuen Palais 10, Building 12, 14469 Potsdam, Germany

2 Centre for Human Movement Sciences, University Medical Centre Groningen, Groningen, The Netherlands

3 Institute of Sport and Sport Science, Albert-LudwigsUniversity of Freiburg, Freiburg, Germany
Data Sources A computerized systematic literature search was performed in the electronic databases PubMed and Web of Science from January 1985 up to January 2015 to capture all articles related to BT in healthy old community-dwelling adults.

Study Eligibility Criteria A systematic approach was used to evaluate the 345 articles identified for initial review. Only RCTs were included if they investigated BT in healthy community-dwelling adults aged $\geq 65$ years and tested at least one behavioral balance performance outcome (e.g., center of pressure displacements during single-leg stance). In total, 23 studies met the inclusionary criteria for review.

Study Appraisal and Synthesis Methods Weighted mean standardized mean differences between subjects $\left(\mathrm{SMD}_{\mathrm{bs}}\right)$ of the intervention-induced adaptations in balance performance were calculated using a random-effects model and tested for an overall intervention effect relative to passive controls. The included studies were coded for the following criteria: training modalities (i.e., training period, training frequency, training volume) and balance outcomes [static/dynamic steady-state (i.e., maintaining a steady position during standing and walking), proactive balance (i.e., anticipation of a predicted perturbation), reactive balance (i.e., compensation of an unpredicted perturbation) as well as balance test batteries (i.e., combined testing of different balance components as for example the Berg Balance Scale)]. Heterogeneity between studies was assessed using $I^{2}$ and $\mathrm{Chi}^{2}$-statistics. The methodological quality of each study was tested by means of the Physiotherapy Evidence Database (PEDro) Scale.

Results Weighted mean $\mathrm{SMD}_{\mathrm{bs}}$ showed that $\mathrm{BT}$ is an effective means to improve static steady-state (mean $\mathrm{SMD}_{\mathrm{bs}}=0.51$ ), dynamic steady-state (mean $\mathrm{SMD}_{\mathrm{bs}}=0.44$ ), proactive (mean $\mathrm{SMD}_{\mathrm{bs}}=1.73$ ), and 
reactive balance (mean $\left.\mathrm{SMD}_{\mathrm{bs}}=1.01\right)$ as well as the performance in balance test batteries (mean $\left.\mathrm{SMD}_{\mathrm{bs}}=1.52\right)$ in healthy older adults. Our analyses regarding dose-response relationships in $\mathrm{BT}$ revealed that a training period of $11-12$ weeks (mean $\mathrm{SMD}_{\mathrm{bs}}=1.26$ ), a frequency of three training sessions per week (mean $\mathrm{SMD}_{\mathrm{bs}}=1.20$ ), a total number of 36-40 training sessions (mean $\mathrm{SMD}_{\mathrm{bs}}=1.39$ ), a duration of a single training session of 31-45 min (mean $\mathrm{SMD}_{\mathrm{bs}}=1.19$ ), and a total duration of 91-120 min of BT per week (mean $\mathrm{SMD}_{\mathrm{bs}}=1.93$ ) of the applied training modalities is most effective in improving overall balance performance. However, it has to be noted that effect sizes for the respective training modalities were computed independently (i.e., modality specific). Because of the small number of studies that reported detailed information on training volume (i.e., number of exercises per training session, number of sets and/or repetitions per exercise, duration of single-balance exercises) dose-response relationships were not computed for these parameters.

Limitations The present findings have to be interpreted with caution because we indirectly compared dose-response relationships across studies using $\mathrm{SMD}_{\mathrm{bs}}$ and not in a single controlled study as it is difficult to separate the impact of a single training modality (e.g., training frequency) from that of the others. Moreover, the quality of the included studies was rather limited with a mean PEDro score of 5 and the heterogeneity between studies was considerable (i.e., $I^{2}=76-92 \%$ ).

Conclusions Our detailed analyses revealed that BT is an effective means to improve proxies of static/dynamic steady-state, proactive, and reactive balance as well as performance in balance test batteries in healthy older adults. Furthermore, we were able to establish effective BT modalities to improve balance performance in healthy older adults. Thus, practitioners and therapists are advised to consult the identified dose-response relationships of this systematic literature review and meta-analysis. However, further research of high methodologic quality is needed to determine (1) dose-response relationships of BT in terms of detailed information on training volume (e.g., number of exercises per training session) and (2) a feasible and effective method to regulate training intensity in BT.

\section{Key Points}

The present systematic review and meta-analysis quantified dose-response relationships of balance training (BT) modalities (i.e., training period, training frequency, training volume) to maximize improvements in balance performance in healthy adults aged 65 years and older.

Our analyses revealed that an effective BT protocol is characterized by the following independently considered training modalities to improve balance performance in healthy older adults: a training period of 11-12 weeks, a frequency of three sessions per week, a total number of 36-40 training sessions, a duration of 31-45 min of a single training session, and a total duration of 91-120 min of BT per week.

Our study provides preliminary evidence-based guidelines on dose-response relationships for practitioners and therapists to increase the efficacy of their BT protocols and to highlight the necessity of studies that incorporate systematically structured BT programs.

\section{Introduction}

Age-related changes in the sensorimotor and neuromuscular system negatively affect performance in static and dynamic postural control even in healthy older adults [1]. Cross-sectional studies highlight that healthy older adults show larger center of pressure displacements (CoP) and sway velocity in bi- and unipedal quiet stances under different conditions (e.g., eyes opened/closed; stable/unstable surface) compared with young adults [2-4]. Critical markers in postural control have been reported in the literature that are associated with an increased risk of falls. For instance, a standing time of $\leq 19 \mathrm{~s}$ in the modified Romberg Test [5], a habitual gait speed of $<1 \mathrm{~m} / \mathrm{s}$ [6], and a duration of $\geq 13.5 \mathrm{~s}$ to complete the Timed-Up-and-Go Test (TUG) [7], are associated with a two- to threefold 
increased risk of falls. The short- and long-term effects of serious fall-related injuries, such as mobility limitations, functional decline, and dependent care, significantly reduce quality of life and increase the risk of early death $[8,9]$.

To mitigate age-related declines in balance performance and prevent falls in old age, a number of studies examined the effects of balance training (BT) over the past years [10, 11]. BT primarily aims at improving postural control by challenging the alignment of the body's center of gravity with regard to the base of support (i.e., feet) [12]. Even though there is evidence from original work that BT is effective in improving measures of postural control and ultimately fall risk and rate in older adults [11, 13-15], there is a void in the literature regarding the aggregation of study findings from original work. This is usually realized by conducting systematic literature reviews and metaanalyses. With regard to the level of evidence, findings from meta-analyses are categorized on the highest evidence level, whereas results from original work [e.g., randomized controlled trials (RCTs)] are classified lower [16].

In a recently published meta-analysis, dose-response relationships were quantified for BT in healthy young adults [17]. These authors quantified training frequency, period, and volume; however, intensity was not quantified because there is no psychometrically sound measure available to describe balance exercise intensity [18]. Findings from the meta-analysis indicated that training modalities mainly behave in an inversed U-shape, indicating optimal as well as below- and above-threshold training stimuli. Compared with healthy young adults, we hypothesize that older adults' BT dose-response relationships may show a shift in inverse U-shapes that is modality specific. Differences in training status/fitness level may demand age-specific BT protocols to achieve optimal training effects. The well-established training principle of progressive overload implies that training modalities (e.g., training frequency, training volume) should correspond to the current training status of a given person to avoid overload of the respective biological system [19]. In addition to training status, advanced age with its associated neuromuscular degenerative processes (e.g., decrease in number and size of particularly type II muscle fibers, loss of sensory and motor neurons) seem to have an impact on the temporal pattern of adaptive processes following training in terms of more time needed for adaptive processes [20]. Based on these premises, there is sufficient justification to determine the age-specific dose-response relationships following BT in older adults.

To the best of our knowledge, there is currently no systematic review and meta-analysis that reported the dose-response relationships of BT training modalities in healthy older adults. Therefore, the objectives of this systematic literature review and meta-analysis are to quantify
BT intervention effects on balance outcomes (static/dynamic steady-state, proactive balance, reactive balance as well as balance test batteries) and to additionally characterize dose-response relationships of BT modalities (i.e., training period, training frequency, training volume) through the analysis of RCTs that could maximize improvements in balance performance in healthy community-dwelling older adults.

\section{Methods}

\subsection{Literature Search}

We performed a computerized systematic literature search in PubMed and Web of Science from January 1985 up to January 2015. Because there is no consistent term for training that incorporates balance exercises [21], we referred to an already established Boolean search syntax that was introduced by Lesinski et al. [17]: (("balance training" OR "neuromuscular training" OR "proprioceptive training" OR "sensorimotor training" OR "instability training" OR "perturbation training") AND (old* OR aged OR senior* OR elder*) NOT (patient* OR disease OR stroke OR Parkinson OR children OR young* OR youth OR adolescents)). In addition, the following filters were activated: text availability: full text; publication dates: 1985/01/01 to 2015/01/31; species: humans, ages: 65+ years; languages: English, German. Further, we checked the reference lists of each included article and we analyzed relevant review articles $[14,22,23]$ in an effort to identify additional suitable studies for inclusion in our analyses.

\subsection{Selection Criteria}

To be eligible for inclusion, studies had to meet the following criteria and report specific experimental characteristics: (a) participants were healthy older adults with a mean age $\geq 65$ years; (b) the study included a BT protocol comprising static/dynamic postural stabilization exercises, and (c) the study tested at least one behavioral balance outcome (e.g., gait speed). Studies with the following features were excluded: (a) non-randomized design; (b) use of only an active control group; (c) inclusion of only one specific type of BT (e.g., balance-related exergames, waterbased training, Tai Chi) or a combined type of BT (e.g., balance and resistance training); (d) used fewer than six sessions (i.e., acute studies); (e) participants' baseline gait speed was $<1.0 \mathrm{~m} / \mathrm{s}$ (in case of a gait speed test) [6], and (f) unavailability of means and standard deviations in the results or if authors did not reply to our request for data. Based on the defined inclusion and exclusion criteria, two 
independent reviewers (ML, UG) screened potentially relevant papers by analyzing titles, abstracts, and full texts of respective articles to elucidate their eligibility.

\subsection{Coding of Studies}

Each study was coded for the following variables: number of participants, sex, and age. We coded BT according to the following training modalities:training period, training frequency, and training volume (i.e., number of training sessions, duration of a single training session, total duration of BT per week, number of exercises per training session, number of sets and/or repetition per exercise, duration of a single BT exercise, e.g., standing time). If BT modalities were not reported in detail, the authors were contacted and missing information was requested. This systematic review will not provide information regarding the influence of training intensity, because to date there is no psychometrically sound measure available to describe balance exercise intensity [18].

According to Shumway-Cook and Woollacott [24], balance control is highly task specific and it has to be separated into different categories: static/dynamic steadystate balance (i.e., maintaining a steady position in sitting, standing, and walking), proactive balance (i.e., anticipation of a predicted disturbance), and reactive balance (i.e., compensation for a disturbance) [25]. In fact, several studies indicated that there are only weak to moderate associations between variables of static/dynamic steadystate, proactive, and reactive balance [26, 27]. With reference to these findings, our analyses focused on different balance outcome categories: (a) static steady-state balance (e.g., CoP displacements during single leg stance), (b) dynamic steady-state balance (e.g., 10-m gait speed test), (c) proactive balance (e.g., Functional-Reach-Test or TUG), (d) reactive balance (e.g., CoP displacements after an unexpected perturbation), and (e) balance test batteries (e.g., Berg Balance Scale). When studies reported multiple variables within one of these outcome categories, only one representative outcome variable was included in the analysis. In the category static steady-state balance, highest priority was given to the single right leg stance with eyes opened. As a proxy for dynamic steady-state balance, gait speed was used. The Functional-Reach-Test was preferably selected as a proxy for proactive balance, and finally for reactive balance, we chose $\mathrm{CoP}$ displacements following a perturbation impulse. The Berg Balance Scale was used as the most prominent balance test battery. If a study used other tests, we decided to include those tests in our quantitative analyses that were most similar in terms of their temporal/spatial structure to the ones described above (e.g., tandem walking).
Because of the limited number of studies that examined the different outcome categories (i.e., static/dynamic steady-state balance, proactive balance, reactive balance), we quantified overall BT dose-response relationships. When studies reported multiple outcome categories, the following decision tree was applied that prioritized the importance of the respective test instrument to assess functional capacity: (a) balance test batteries, (b) dynamic steady-state balance, (c) reactive balance, (d) proactive balance, and (e) static steady-state balance. If a study implemented an exercise progression scheme over the training period, the mean number of exercises per training session, sets and/or repetitions per exercise, and duration of balance exercises were calculated.

\subsection{Assessment of Methodological Quality and Statistical Analyses}

The Physiotherapy Evidence Database (PEDro) Scale was used to assess the methodological quality of all eligible intervention studies. The PEDro Scale rates internal study validity and it rates the presence of statistical replicable information on a scale from 0 to 10 with $\geq 6$ representing a cut-off score for high-quality studies [28].

To verify the effectiveness of BT on a balance outcome measures, we computed the within-subject standardized mean difference $\left[\mathrm{SMD}_{\mathrm{ws}}=([\right.$ mean pre-value - mean post-value]/SD pre-value)] and the between-subject standardized mean difference $\left[\mathrm{SMD}_{\mathrm{bs}}=([\right.$ mean post-value intervention group - mean post-value control group]/ pooled variance)]. We adjusted the $\mathrm{SMD}_{\mathrm{bs}}$ for the respective sample size: $g=\left(1-\frac{3}{4 N_{i}-9}\right)$, where $N_{i}$ is the number of subjects [29, 30]. In addition, included studies were weighted according to the magnitude of the respective standard error using Review Manager version 5.3.4 (Copenhagen: The Nordic Cochrane Centre, The Cochrane Collaboration, 2008). A random-effects meta-analysis model was applied to compute the weighted mean $\mathrm{SMD}_{\mathrm{bs}}$ in Review Manager version 5.3.4. Depending on the respective outcome measure (i.e., sway path vs. time of single leg stance), $\mathrm{SMD}_{\mathrm{ws}} / \mathrm{SMD}_{\mathrm{bs}}$ can be negative or positive. To improve readability, we reported positive changes in outcomes $\left(\mathrm{SMD}_{\mathrm{ws}}\right)$ and superiority of $\mathrm{BT}$ compared with the control $\left(\mathrm{SMD}_{\mathrm{bs}}\right)$ with a positive $\mathrm{SMD}_{\mathrm{ws}} / \mathrm{SMD}_{\mathrm{bs}}$. The calculation of $\mathrm{SMD}_{\mathrm{ws}} / \mathrm{SMD}_{\mathrm{bs}}$ allows us to conduct a systematic and quantitative evaluation of the different BT modalities including a large number of studies and it helps to determine whether a difference is of practical concern. According to Cohen [31], effect size values of $0.00 \leq 0.49$ indicate small, of $0.50 \leq 0.79$ indicate medium, and of $\geq 0.80$ indicate large effects. 
Fig. 1 Flow chart illustrating the different phases of the search and study selection

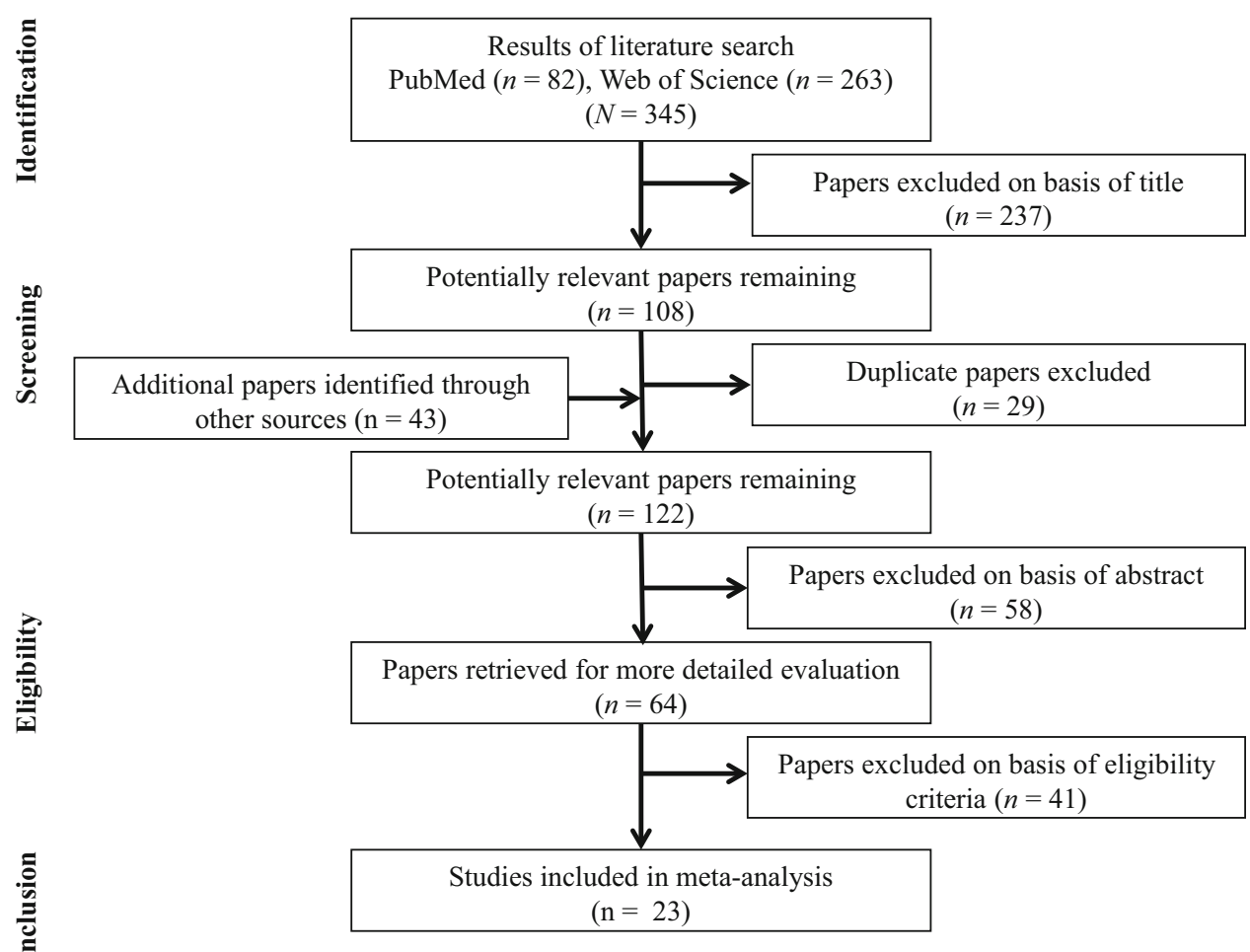

state, proactive, and reactive balance exercises on stable/ unstable surfaces (e.g., BOSU ${ }^{\circledR}$ ball, tilt board, trampoline, rocker board, DynaDisc ${ }^{\circledR}$, wobble board, foam mat, balance platform) and balance systems (e.g., Biodex Balance System) with eyes opened or closed. Moreover, many BT protocols contained exercises that were related to activities of daily living, such as obstacle walking. Twelve of 23 studies reported information on progression during training in terms of an increase in level of difficulty of BT. Most studies $(n=12)$ used static steady-state balance tests as the outcome parameter (e.g., center of pressure displacements during unipedal stance) to assess training effects [32-43], seven studies used proxies of dynamic steadystate balance (e.g., gait speed) [34, 35, 38, 42, 44-46], seven studies used proactive balance tests (e.g., Functionalreach-test) [32, 37, 42, 44, 47-49], five studies applied a reactive balance test (e.g., Push-and-release-test) [44, 50 53], and another five studies used a balance test battery (e.g., Berg Balance Scale) [32, 38, 47, 48, 54].

\subsection{Methodological Quality of the Included Trials}

The quality of the included studies can be classified as weak, because 17 out of 23 studies did not reach the predetermined cut-off value of 6 on the PEDro Scale (Table 2) [28]. For all investigated studies, a median PEDro score of 5 (range 3-8) was detected. Additionally, only a few 


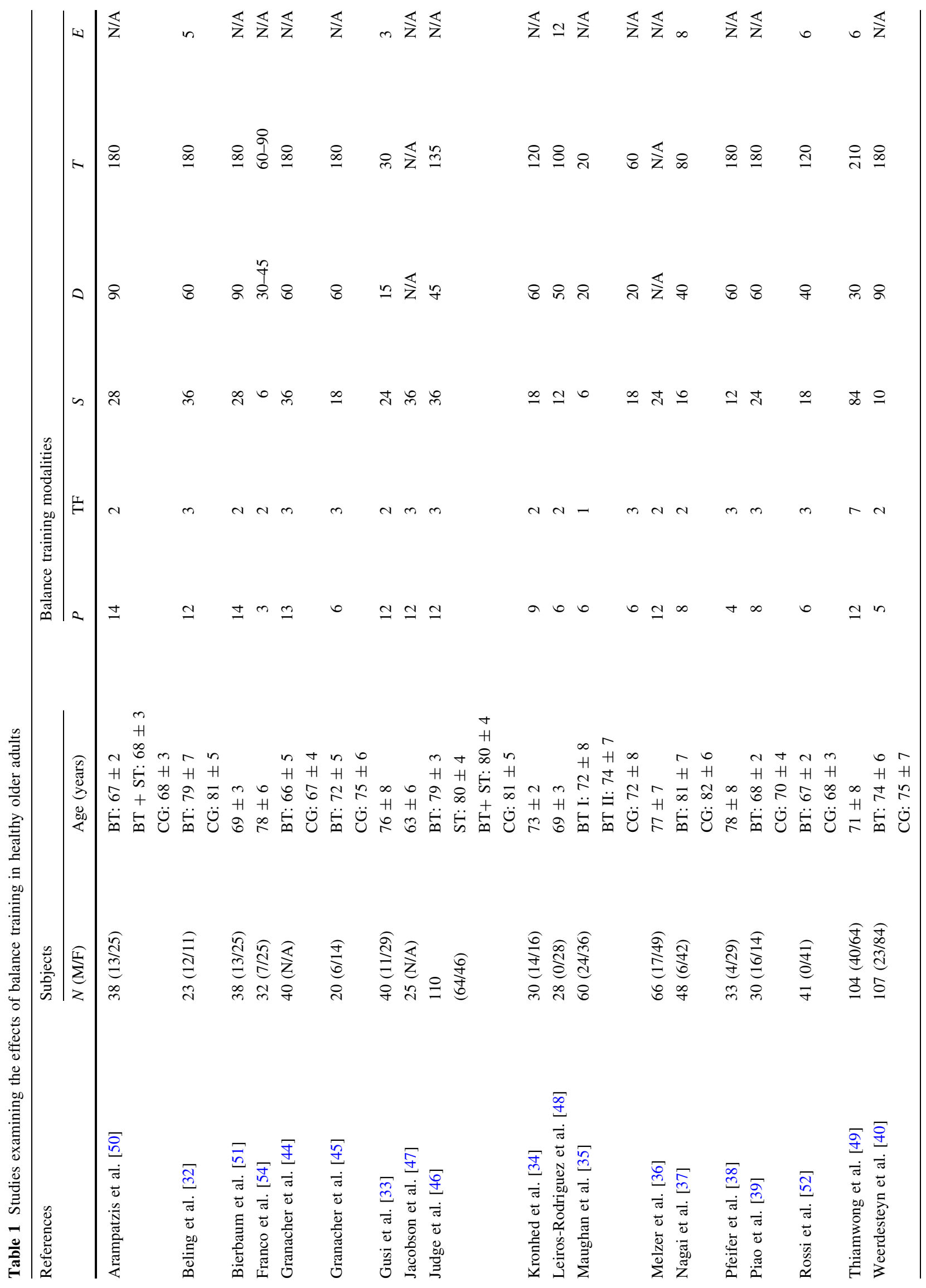




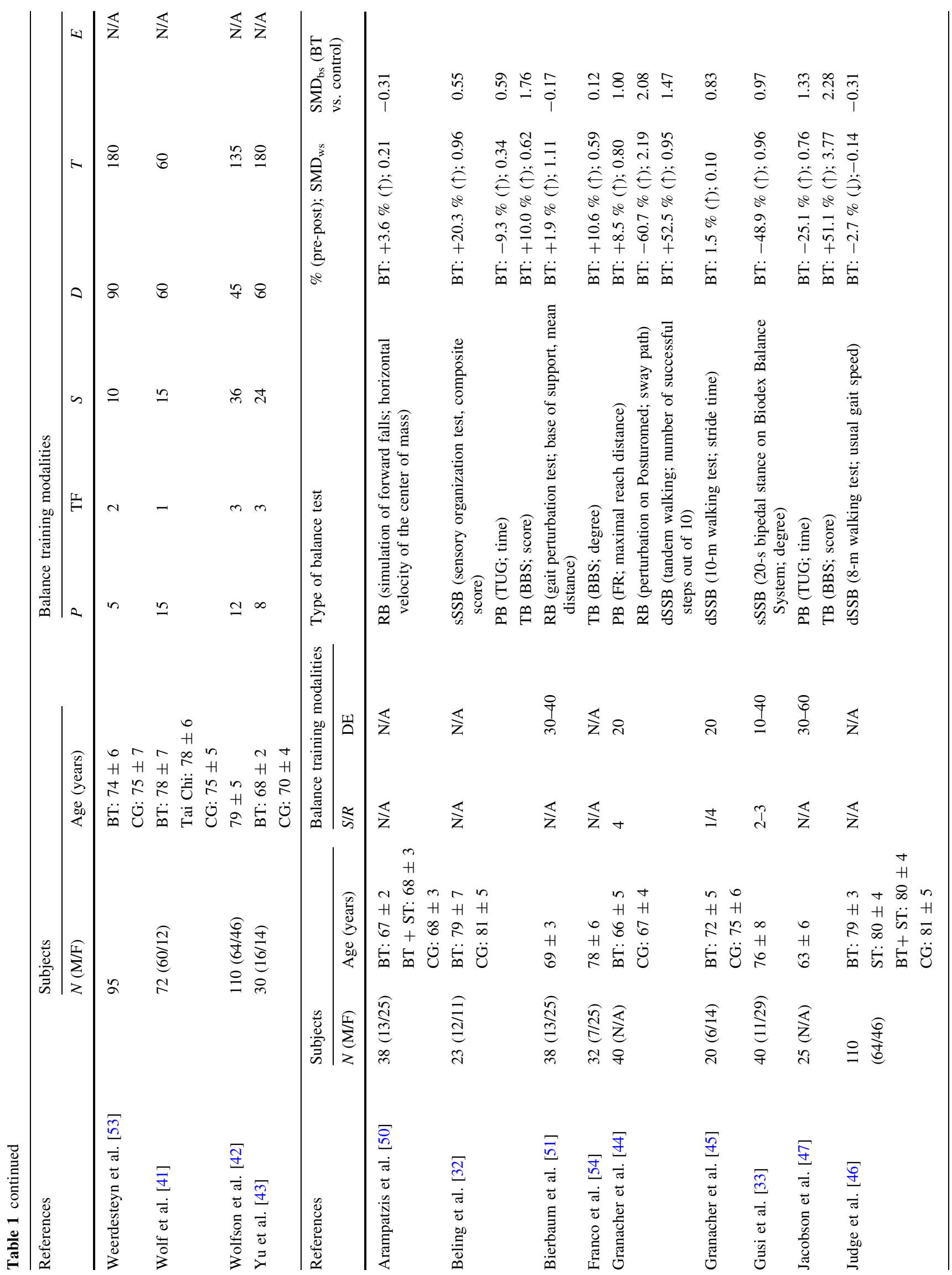




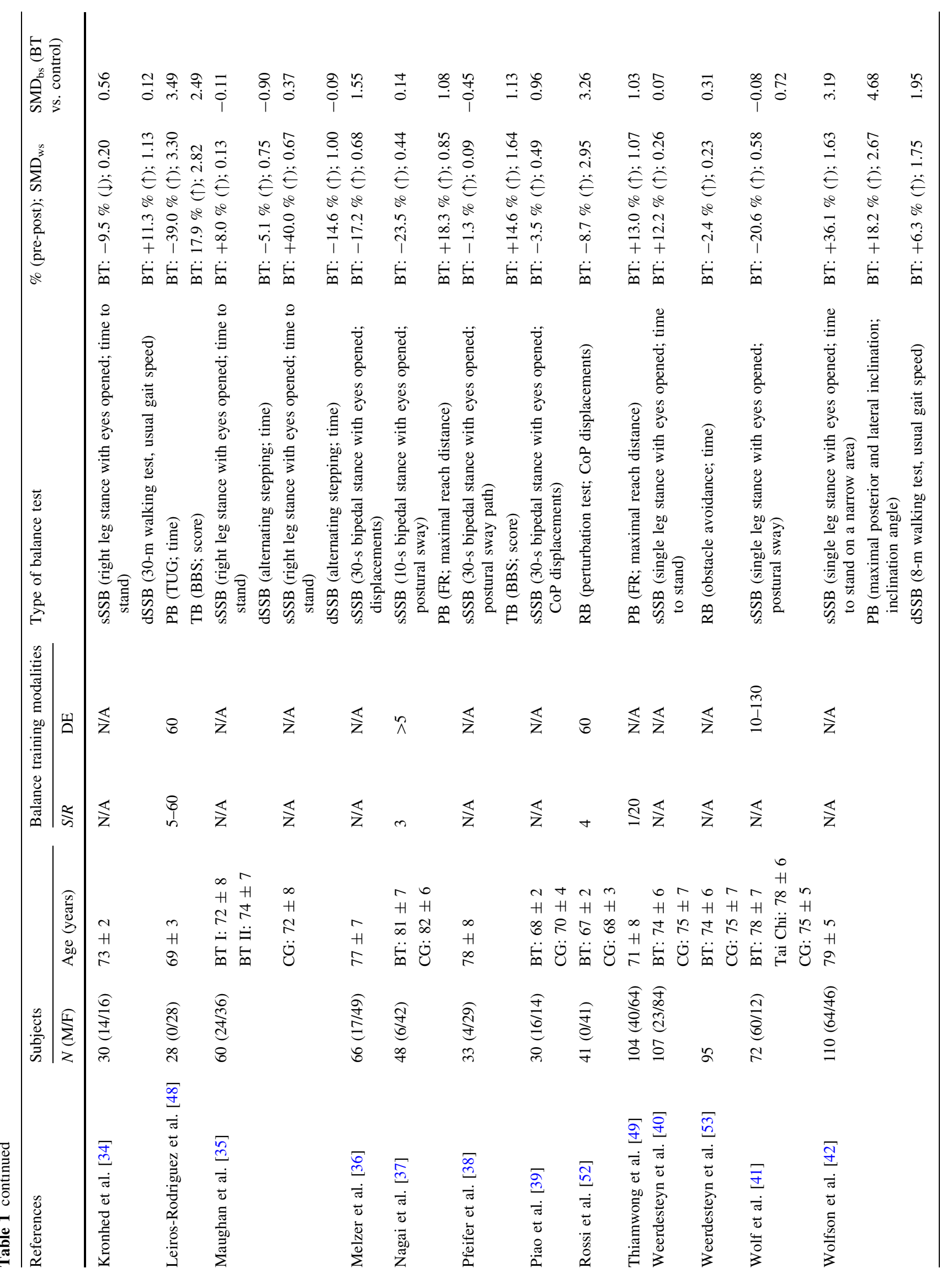




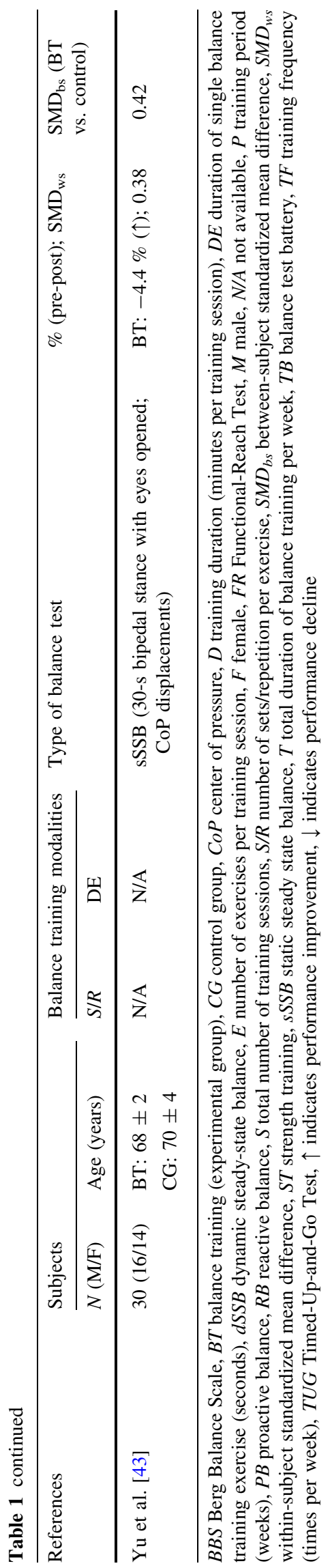

studies reported detailed information regarding the entire BT protocol. Limited and/or incomplete information was specifically reported for training volume (e.g., number of exercises per training session, number of sets per exercise, duration of a single BT exercise) (Table 1).

\subsection{Effectiveness of BT}

Figures 2, 3, 4, 5, and 6 illustrate the effects of BT vs. a passive control group on proxies of static/dynamic steadystate, proactive, and reactive balance as well as for balance test batteries. Weighted mean $\mathrm{SMD}_{\mathrm{bs}}$ amounted to 0.51 for measures of static steady-state balance (12 studies; $\left.I^{2}=83 \%, \mathrm{Chi}^{2}=69.95, d f=12, p<0.001\right), 0.44$ for variables of dynamic steady-state balance (7 studies; $\left.I^{2}=88 \%, \mathrm{Chi}^{2}=57.16, d f=7, p<0.001\right), 1.73$ for variables of proactive balance ( 7 studies; $I^{2}=86 \%$, $\left.\mathrm{Chi}^{2}=41.90, d f=6, p<0.001\right), 1.01$ for variables of reactive balance ( 5 studies; $I^{2}=92 \%, \mathrm{Chi}^{2}=52.95$, $d f=4, p<0.001)$, and 1.52 for balance test batteries ( 5 studies; $I^{2}=76 \%, \mathrm{Chi}^{2}=16.46, \quad d f=4, \quad p<0.01$ ), indicating small to large effects.

\subsection{Dose-response relationships}

Figures 7, 8, 9, 10, and 11 present the overall dose-response relationships (all included studies). Because of the limited number of studies that examined proxies of dynamic steady-state balance, proactive balance, reactive balance, and balance test batteries, specific dose-response relationships were quantified for static steady-state balance only (Table 3). Even though a few authors responded to our inquiries and sent study-specific detailed information on BT modalities, we were not able to quantify dose-response relationships for certain training modalities (i.e., number of exercises per training session, number of sets and/or repetitions per exercise, duration of single BT exercises). Of note, authors did not use a standardized set of BT modalities (e.g., sets, repetitions) to describe the program. Instead, some authors provided time constraints for different BT exercises (e.g., subjects had 10 min time to train different balance exercises with different difficulty level).

\subsubsection{Training Period}

Figure 7 illustrates the overall dose-response relationship for the parameter 'training period'. Our analyses revealed that a training period of 11-12 weeks produced the largest effects on both overall balance performance (mean $\mathrm{SMD}_{\mathrm{bs}}=1.26 ; 23$ studies) as well as for more specific measures of static steady-state balance (mean $\mathrm{SMD}_{\mathrm{bs}}=1.54 ; 12$ studies). 


$$
\text { L }
$$




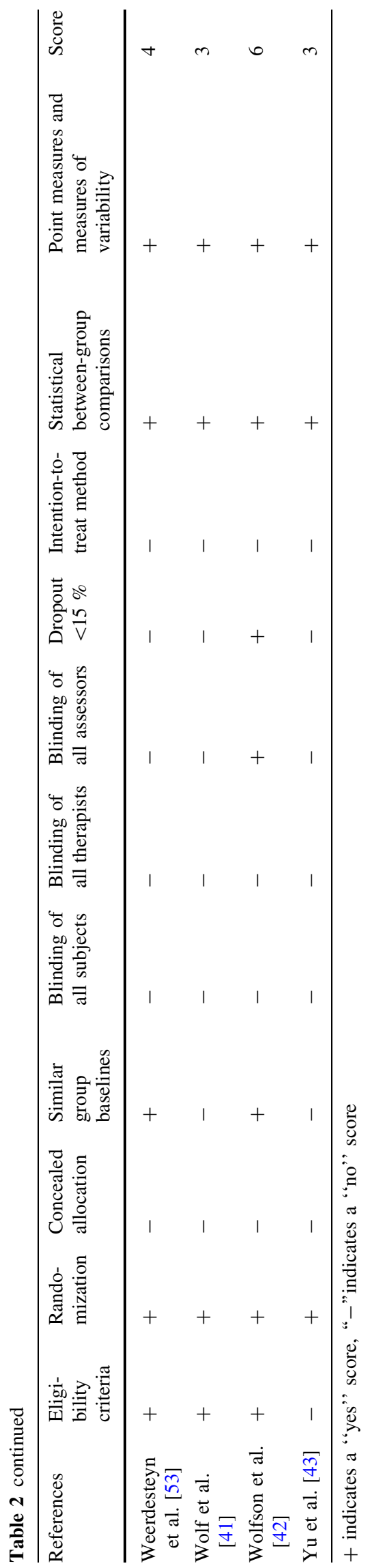

\subsubsection{Training Frequency}

Figure 8 presents the overall dose-response relationship regarding training frequency. A BT frequency of three sessions/week resulted in the largest effects for improving both measures of overall balance performance (mean $\mathrm{SMD}_{\mathrm{bs}}=1.20 ; 23$ studies) as well as for more specific measures of static steady-state balance (mean $\mathrm{SMD}_{\mathrm{bs}}=0.81 ; 12$ studies).

\subsubsection{Training Volume (Number of Training Sessions During the Training Period)}

Figure 9 displays the overall dose-response relationship regarding the total number of training sessions. Our findings indicate that a total number of 36-40 training sessions is most effective in improving both overall balance performance (mean $\mathrm{SMD}_{\mathrm{bs}}=1.39 ; 23$ studies) as well as for more specific measures of static steady-state balance (mean $\mathrm{SMD}_{\mathrm{bs}}=1.87 ; 12$ studies).

\subsubsection{Training Volume (Duration of a Single Training Session)}

Figure 10 presents the overall dose-response relationship regarding the duration of single training sessions. Our findings revealed that a duration of 31-45 minis most effective to improve overall balance performance (mean $\mathrm{SMD}_{\mathrm{bs}}=1.19 ; 21$ studies) as well as for more specific measures of static steady-state balance (mean $\mathrm{SMD}_{\mathrm{bs}}=1.64 ; 11$ studies $)$.

\subsubsection{Training Volume (Total Duration of Training Per Week)}

Figure 11 displays the overall dose-response relationship regarding the total duration of training per week. Our findings indicate that a total duration of 91-120 min of BT per week is most effective in improving overall balance performance (mean $\mathrm{SMD}_{\mathrm{bs}}=1.93 ; 21$ studies). In terms of improving proxies of static steady-state balance a total duration of $121-150 \mathrm{~min}\left(\mathrm{SMD}_{\mathrm{bs}}=3.19\right.$; one study only) of BT per week produced the largest effects.

\section{Discussion}

This is the first systematic literature review and metaanalysis to examine the overall effects of BT on proxies of balance performance and to characterize and quantify the dose-response relationships of BT modalities (i.e., training period, training frequency, training volume) leading to balance improvements in healthy older adults. Analyses of 


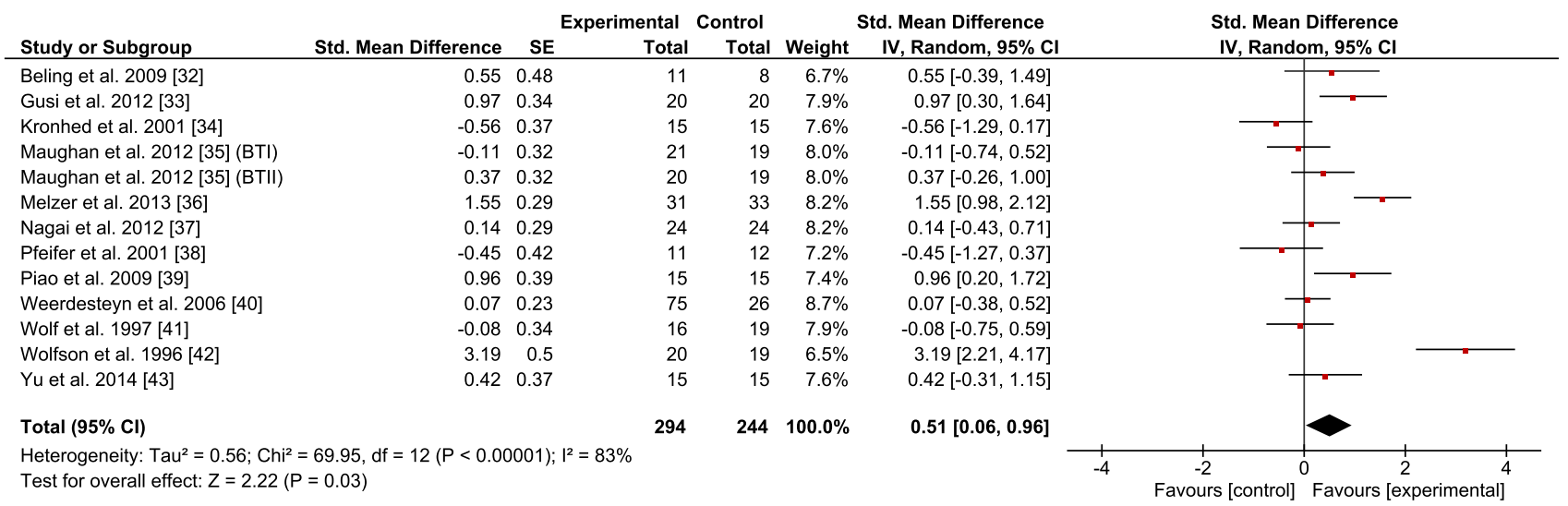

Fig. 2 Effects of balance training (experimental) vs. control on measures of static steady-state balance. $C I$ confidence interval, $S E$ standard error, Std. standard, $I V$ inverse variance

\begin{tabular}{|c|c|c|c|c|c|c|c|c|c|}
\hline Study or Subgroup & Std. Mean Difference & \multicolumn{2}{|r|}{ Experimental } & $\begin{array}{l}\text { Control } \\
\text { Total }\end{array}$ & \multicolumn{2}{|r|}{ Std. Mean Difference } & \multicolumn{2}{|c|}{$\begin{array}{l}\text { Std. Mean Difference } \\
\text { IV, Random, } 95 \% \mathrm{CI}\end{array}$} & \\
\hline Granacher et al. 2009 [44] & 1.47 & 0.36 & 20 & 20 & $12.5 \%$ & $1.47[0.76,2.18]$ & & $\square$ & \\
\hline Granacher et al. 2010 [54] & 0.83 & 0.47 & 11 & 9 & $11.4 \%$ & $0.83[-0.09,1.75]$ & & & \\
\hline Judge et al. 1994 [46] & -0.31 & 0.27 & 28 & 27 & $13.2 \%$ & $-0.31[-0.84,0.22]$ & & & \\
\hline Kronhed et al. 2001 [34] & 0.12 & 0.37 & 15 & 15 & $12.4 \%$ & $0.12[-0.61,0.85]$ & & 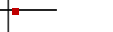 & \\
\hline Maughan et al. 2012 [35] (BTI) & -0.9 & 0.33 & 21 & 19 & $12.7 \%$ & $-0.90[-1.55,-0.25]$ & & & \\
\hline Maughan et al. 2012 [35] (BTII) & -0.09 & 0.32 & 20 & 19 & $12.8 \%$ & $-0.09[-0.72,0.54]$ & & & \\
\hline Pfeifer et al. 2001 [38] & 0.57 & 0.38 & 15 & 14 & $12.3 \%$ & $0.57[-0.17,1.31]$ & & & \\
\hline Wolfson et al. 1996 [42] & 1.95 & 0.33 & 27 & 27 & $12.7 \%$ & $1.95[1.30,2.60]$ & & & \\
\hline Total $(95 \% \mathrm{Cl})$ & & & 157 & 150 & $100.0 \%$ & $0.44[-0.24,1.13]$ & & & \\
\hline $\begin{array}{l}\text { Heterogeneity: } \mathrm{Tau}^{2}=0.85 ; \mathrm{Chi}^{2} \\
\text { Test for overall effect: } Z=1.27\end{array}$ & $\begin{array}{l}=57.16, \mathrm{df}=7(\mathrm{P}<0.000 \\
=0.20)\end{array}$ & 001); $1^{2}$ & $\left.\right|^{2}=88 \%$ & & & & $\begin{array}{c}1 \\
-2 \\
\text { Favours [control] }\end{array}$ & ${ }^{0}$ Favours [e & 2 \\
\hline
\end{tabular}

Fig. 3 Effects of balance training (experimental) vs. control on measures of dynamic steady-state balance. $C I$ confidence interval, $S E$ standard error, $S t d$. standard, $I V$ inverse variance

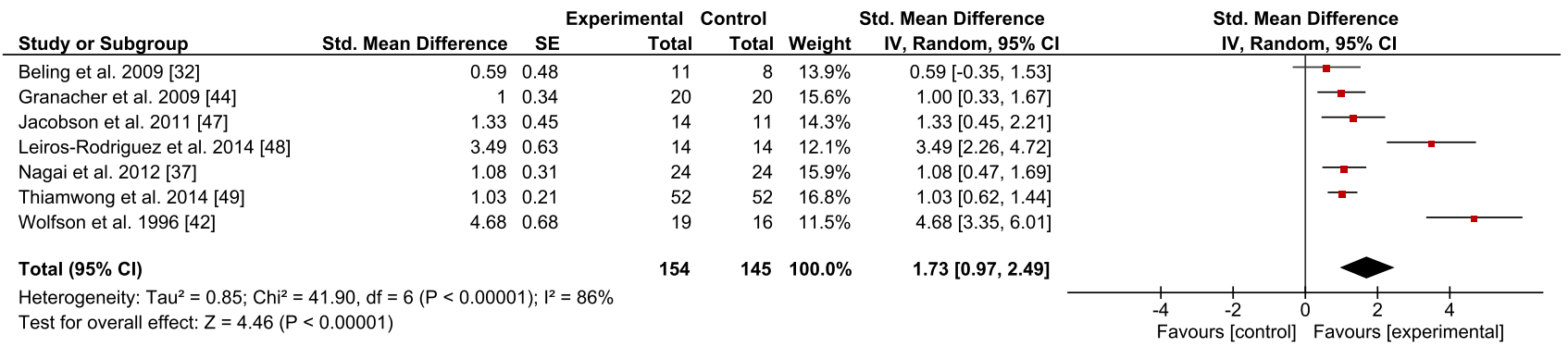

Fig. 4 Effects of balance training (experimental) vs. control on measures of proactive balance. $C I$ confidence interval, $S E$ standard error. Std. standard, $I V$ inverse variance

BT data from 23 RCTs revealed that BT is an effective method to improve healthy older adults' balance performance. However, the nature of these responses is nearly identical to those reported previously in young adults (Table 3). Against our hypothesis, the results raise the possibility that age does not affect BT parameters known to produce adaptations in static and dynamic measures of balance. We discuss these findings by interpreting the general effects of BT with reference to the already available literature and by taking potential age-specific dose-response relationships into account.

\subsection{Effectiveness of Balance Training}

A number of reviews and meta-analyses already examined the effects of different fall prevention programs in older adults $[11,14,55-59]$ and revealed that among others BT is recommended if the main goal is to reduce risk and rate 


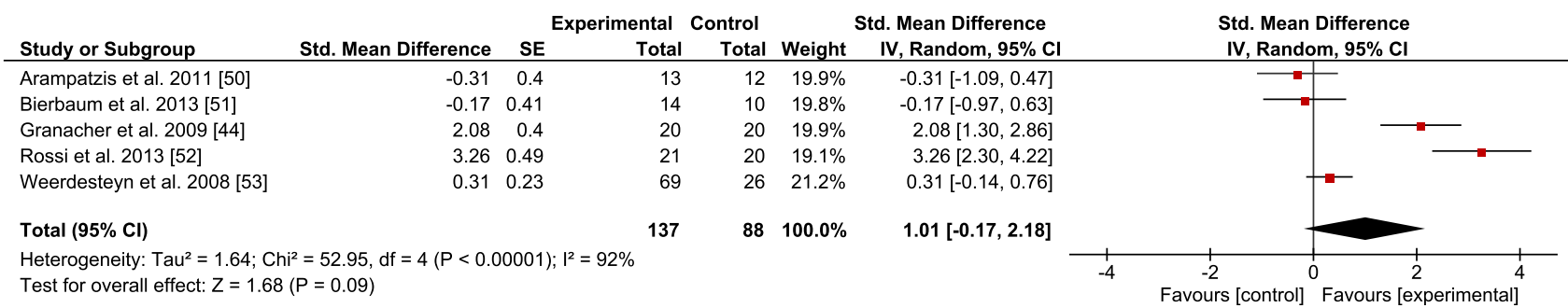

Fig. 5 Effects of balance training (experimental) vs. control on measures of reactive balance. $C I$ confidence interval, $S E$ standard error, $S t d$. standard, $I V$ inverse variance

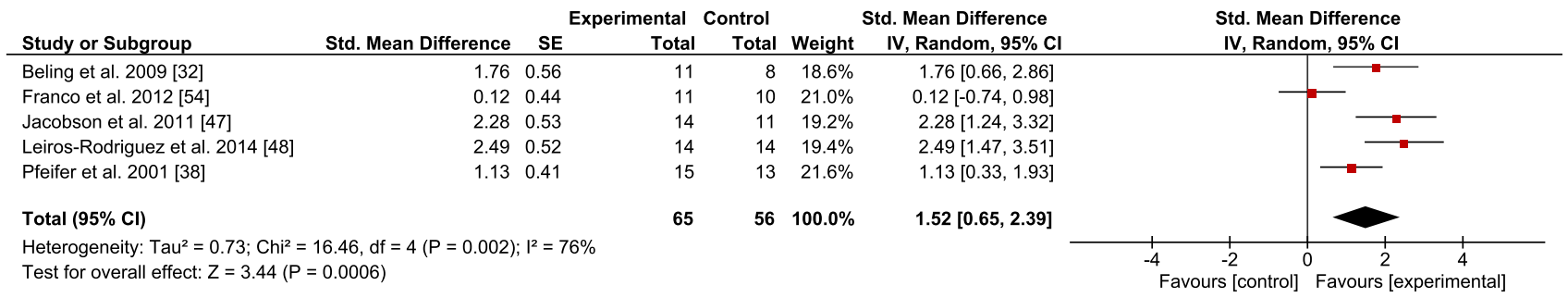

Fig. 6 Effects of balance training (experimental) vs. control on performance in balance test batteries, $C I$ confidence interval, $S E$ standard error, Std. standard, $I V$ inverse variance

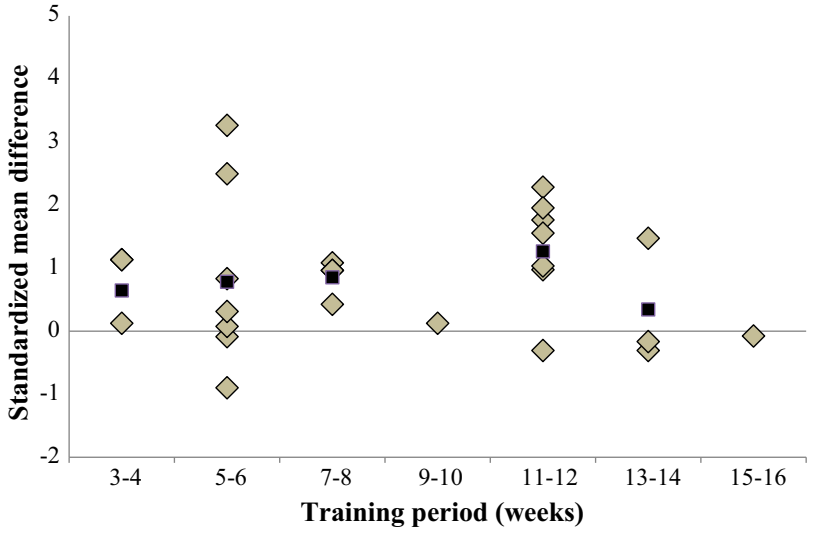

Fig. 7 Dose-response relationships of training period on overall balance performance. Each filled gray diamond illustrates betweensubject standardized mean difference $\left(\mathrm{SMD}_{\mathrm{bs}}\right)$ per single study with passive control. Filled black squares represent weighted mean $\mathrm{SMD}_{\mathrm{bs}}$ of all studies

of falls in older adults $[11,14,55,56,58]$. However, there is no systematic review and meta-analysis available that examined the effects of BT on different measures of balance performance (i.e., static/dynamic steady-state balance, proactive balance, reactive balance, balance test batteries). Our analyses showed that BT is effective in improving measures of static/dynamic steady-state, proactive, and reactive balance as well as performance in balance test batteries in healthy old age. Thereby, the effects of BT on measures of static/dynamic steady-state balance are small to medium compared with large effects on proxies of

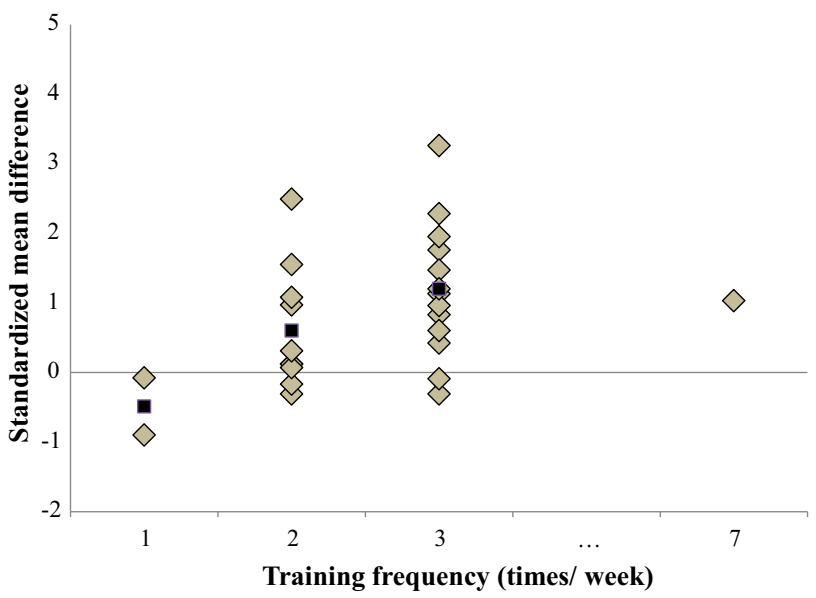

Fig. 8 Dose-response relationships of training frequency on overall balance performance. Each filled gray diamond illustrates betweensubject standardized mean difference $\left(\mathrm{SMD}_{\mathrm{bs}}\right)$ per single study with passive control. Filled black squares represent weighted mean $\mathrm{SMD}_{\mathrm{bs}}$ of all studies

proactive and reactive balance as well as on performance in balance test batteries. Potential ceiling effects may account for the lower effectiveness of BT regarding static/dynamic steady-state balance. Another factor contributing to the small to medium effect sizes is the large difference between the complex temporal and spatial structure of the BT stimuli delivered through the BT programs and the nonspecific simple structure of the static balance tests. In terms of dynamic steady-state balance five of seven studies examined habitual gait speed pre- and post-BT. The 


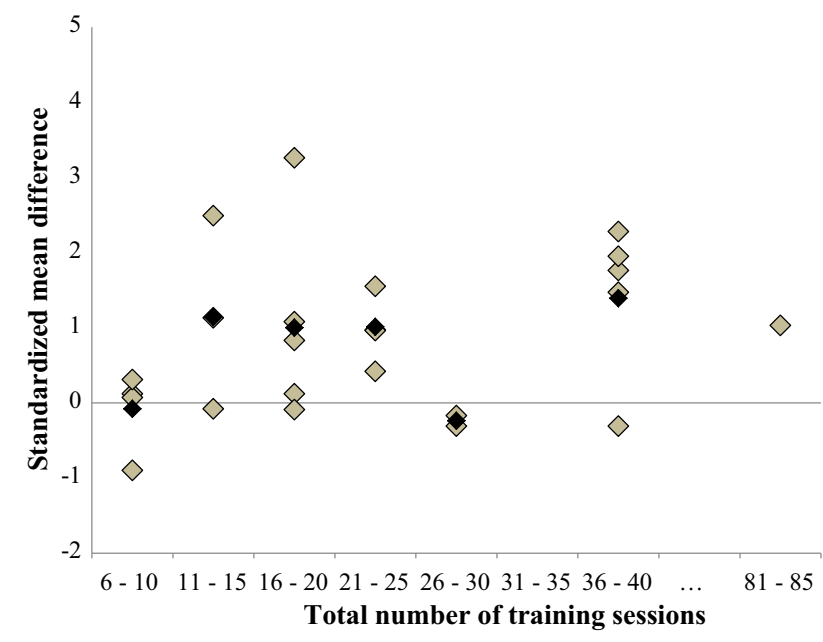

Fig. 9 Dose-response relationships of total number of training sessions on overall balance performance. Each filled gray diamond illustrates between-subject standardized mean difference $\left(\mathrm{SMD}_{\mathrm{bs}}\right)$ per single study with passive control. Filled black squares represent weighted mean $\mathrm{SMD}_{\mathrm{bs}}$ of all studies

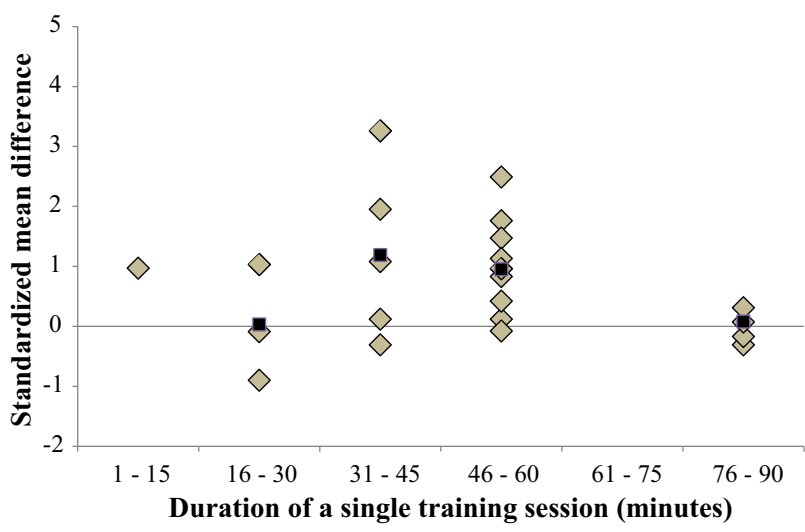

Fig. 10 Dose-response relationships of the duration of a single training session on overall balance performance. Each filled gray diamond illustrates between-subject standardized mean difference $\left(\mathrm{SMD}_{\mathrm{bs}}\right)$ per single study with passive control. Filled black squares represent weighted mean $\mathrm{SMD}_{\mathrm{bs}}$ of all studies

subjects mean baseline gait speed $(1.3 \mathrm{~m} / \mathrm{s})$ can be classified as high and is indicative that the included subjects were not mobility limited and had a low risk of falls [60]. Despite the fact that the weighted mean $\mathrm{SMD}_{\mathrm{bs}}$ of 0.44 was small for proxies of dynamic steady-state balance, the absolute increase in gait speed of $0.07 \mathrm{~m} / \mathrm{s}$ represents a small but meaningful improvement in gait speed, particularly for healthy older adults $[60,61]$.

\subsection{Dose-response relationships following balance training}

The scrutinized studies used a broad range of training periods (4-15 weeks), frequencies (1-7 times/week),

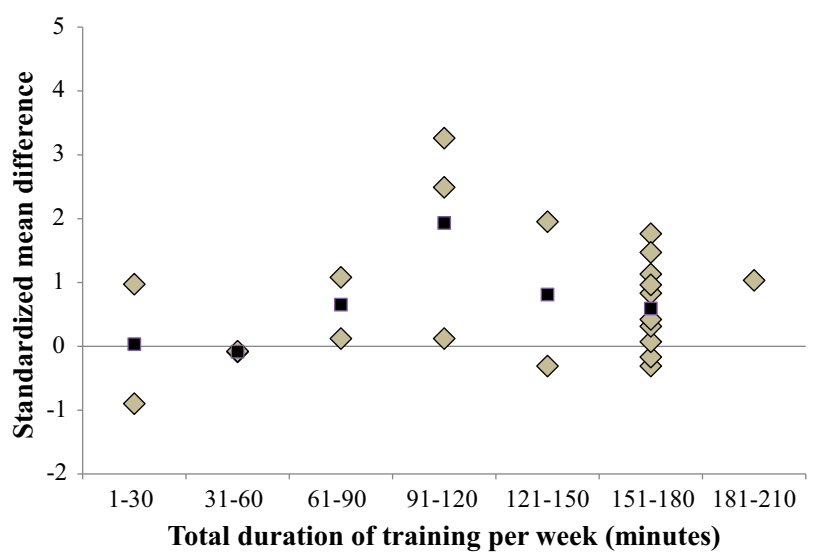

Fig. 11 Dose-response relationships of the total duration of balance training per week on overall balance performance. Each filled gray diamond illustrates between-subject standardized mean difference $\left(\mathrm{SMD}_{\mathrm{bs}}\right)$ per single study with passive control. Filled black squares represent mean $\mathrm{SMD}_{\mathrm{bs}}$ of all studies

number of total training sessions (6-84 training sessions), durations of single training sessions (15-90 $\mathrm{min} / \mathrm{session})$, and total durations of BT per week (20-210 min/week). Both the general as well as the specific dose-response relationships for overall balance performance and for measures of static steady-state balance revealed that a training period of 11-12 weeks, a frequency of three sessions per week, a total number of 36-40 training sessions, a duration of a single training session of $31-45 \mathrm{~min}$, and a total duration of 91-120 min of BT per week is most effective to improve balance. Given that only a few included studies reported detailed information on training volume (i.e., the number of exercises per training session, number of sets and/or repetitions per exercise, duration of single BT exercises) as well as examined the effects of BT on measures of dynamic steady-state balance, proactive balance, and reactive balance as well as balance test batteries, we were not able to quantify dose-response relationships for each specific outcome category.

\subsubsection{Training Period}

Our analysis illustrates that BT lasting between 11 and 12 weeks is most effective in enhancing both overall balance performance (mean $\mathrm{SMD}_{\mathrm{bs}}=1.26 ; 23$ studies) and static steady-state balance (mean $\mathrm{SMD}_{\mathrm{bs}}=1.54 ; 12$ studies). Figure 7 illustrates that less than 11 weeks of training resulted in lower effects on balance performance. This result is in accordance with Lesinski et al. [17], who quantified the dose-response relationships of BT in young adults (i.e., 18-40 years). Our findings agree with those for young adults in as much as a training period of at least 11-12 weeks is more effective to improve static steadystate balance as compared with shorter training periods 
Table 3 Dose-response relationships for balance training in healthy older adults

\begin{tabular}{|c|c|c|c|}
\hline \multirow[t]{3}{*}{ Training modalities } & \multicolumn{3}{|c|}{ Results/most effective dose } \\
\hline & \multicolumn{2}{|c|}{ Healthy older adults } & \multirow{2}{*}{$\begin{array}{l}\text { Healthy young adults [17] } \\
\text { Static steady-state balance }\end{array}$} \\
\hline & Overall balance & Static steady-state balance & \\
\hline Training period (weeks) & $11-12$ & $11-12$ & $11-12$ \\
\hline Training frequency (times per week) & 3 & 3 & 3 \\
\hline Number of training sessions & $36-40$ & $36-40$ & $16-19 ; 36-39^{\mathrm{a}}$ \\
\hline Duration of a single training session (min) & $31-45$ & $31-45$ & $11-15^{\mathrm{b}}$ \\
\hline Total duration of BT per week (min) & $91-120$ & $121-150$ (only one study) & N/A \\
\hline Number of exercises per training session & N/A & N/A & 4 \\
\hline Number of sets/reps per exercise & N/A & N/A & 2/N/A \\
\hline Duration of a single balance exercise (s) & N/A & N/A & $21-40$ \\
\hline
\end{tabular}

It has to be noted that training modalities were considered independently

$B T$ balance training, N/A not available, reps repetitions

a Almost identical effect sizes (1.12 vs. 1.09)

${ }^{\mathrm{b}}$ Fourteen out of fifteen studies of BT contained no warm-up and/or cool-down phase and thus were shorter in overall training time than single BT sessions in older adults

(Table 3). Therefore, it seems that there is no age-effect in terms of training period because both meta-analyses observed largest effects when conducting BT for 11-12 weeks. Given that only few studies examined BT periods of more than 12 weeks, this result is preliminary.

A previous review that examined the efficacy of BT to reduce falls [14] concluded that training interventions that involved higher dose of exercise $(>50 \mathrm{~h})$ were more effective to reduce falls and recommended at least $2 \mathrm{~h}$ of training per week for a training period of 6 months. This might indicate that a BT period of more than 12 weeks could be even more effective in improving overall balance performance.

It is of interest to know whether training-induced adaptations are stable over time or whether they decline during detraining. In this regard, a previous study [62] investigated the effects of static/dynamic BT under singleand dual-task conditions during unipedal stance performance with eyes opened and closed in healthy elderly fallers ( $n=8$; mean age $71 \pm 5$ years) and non-fallers ( $n=8$; mean age $68 \pm 5$ years). A 3 -month detraining period resulted in a significant decline in unipedal stance performance in fallers and non-fallers. Likewise, Rossi et al. [52] shows that perturbation-based BT for 6 weeks improved neuromuscular responses (e.g., reaction time) following perturbations (i.e., simulation of sudden forward and backward balance loss due to a sliding apparatus) in community-dwelling older women $(n=41$; mean age $67 \pm 3$ years). However, the training-induced gains were not stable but declined after 6 weeks of detraining. With reference to the studies of Toulotte et al. [62] and Rossi et al. [52] and the recommendation of Sherrington et al.
[14], we advise to conduct BT on a permanent basis to counteract age-related declines in balance performance.

\subsubsection{Training Frequency}

Our analysis revealed that a training frequency of three sessions per week is more effective to improve overall balance performance (mean $\mathrm{SMD}_{\mathrm{bs}}=1.20 ; 23$ studies) and static steady-state balance (mean $\mathrm{SMD}_{\mathrm{bs}}=0.81 ; 12$ studies) compared with BT comprising one to two sessions per week. In an intervention study, Maughen et al. [35] examined the specific effects of BT frequency on proxies of static/dynamic steady-state balance in healthy, physically active older adults ( $n=60$; mean age $73 \pm 8$ years). The authors were able to show that the group that conducted three sessions per week produced larger performance increases after 6 weeks of BT as compared with the group that executed BT once a week. However, the results from this study have to be interpreted with caution because it might be confounded by a higher number of total training sessions (18 vs. 6 training sessions). Still, our findings are confirmed by the recently published systematic review and meta-analysis on dose-response relationships of BT in young healthy adults [17] (Table 3). It appears that there is no age effect in terms of training frequency because both meta-analyses observed largest effects when conducting BT three times per week.

\subsubsection{Training Volume (Number of Training Sessions)}

Concerning the number of training sessions, our analysis revealed that an overall number of 36-40 training sessions 
produced the largest effects in terms of overall balance performance (mean $\mathrm{SMD}_{\mathrm{bs}}=1.39 ; 23$ studies) and static steady-state balance (mean $\mathrm{SMD}_{\mathrm{bs}}=1.87 ; 12$ studies). However, given that only one study examined the effects of more than $40 \mathrm{BT}$ sessions, the result is preliminary. Sherrington et al. [14] showed that there are greater benefits from a higher dose of exercise $(>50 \mathrm{~h})$ that challenges balance and aims at reducing the risk of falls. Therefore, it might be that BT programs should contains at least 36-40 training sessions but indeed will obtain advantages of more than 40 training sessions in terms of training effects on overall balance performance.

\subsubsection{Training Volume (Duration of a Single Training Session and Total Duration of Training per Week)}

In terms of BT durations, our analyses highlighted that $31-45$ min of a single BT session (mean $\mathrm{SMD}_{\mathrm{bs}}=1.19$; 22 studies) and 91-120 min of total BT per week (mean $\mathrm{SMD}_{\mathrm{bs}}=1.93 ; 21$ studies) seem to be most effective to improve overall balance performance. For improving proxies of static steady-state balance our analysis revealed that 31-45 min of a single BT session (mean $\mathrm{SMD}_{\mathrm{bs}}=1.64 ; \quad 11 \quad$ studies) and $121-150 \mathrm{~min}$ $\left(\mathrm{SMD}_{\mathrm{bs}}=3.19\right.$; one study only $)$ of total $\mathrm{BT}$ per week produced the largest effects.

In accordance with the dose-response relationship of BT in young adults [17], there seems to be an inverse U-shaped relation between the effectiveness of $\mathrm{BT}$ and the duration of a single training session in old age. However, peak mean $\mathrm{SMD}_{\mathrm{bs}}$ values shifted to the right, to longer durations (i.e., 31-45 min) in older adults compared with young adults (i.e., 11-15 min). This shift in peak mean $\mathrm{SMD}_{\mathrm{bs}}$ can most likely be explained by the fact that most BT programs conducted in young adults (particularly in athletes) were either performed immediately before or after the sportspecific training. In older adults, training sessions consisted of BT only, included warm-ups and cool downs, and thus took more time. Taking this into account, the net balance training time appears to be almost similar in healthy older adults compared with young adults. Of note, our detailed analyses revealed that BT durations of more than $60 \mathrm{~min}$ produce no additional training effects in older adults. In fact, it seems to be more effective to split the total duration of BT per week (i.e., about 91-120 min) into more (i.e., three or more per week) and shorter (i.e., about 31-45 min) single training sessions, instead of longer single training sessions (i.e., $\geq 60 \mathrm{~min}$ ) that are conducted one-two times per week only.

Given that only a few studies reported the number of exercises per training session, the number of sets and/or repetitions per exercise, and the duration of single-balance exercises, dose-response relationships were not computed for these training modalities. In addition, there is no methodological sound approach available in the literature on how to properly assess intensity during BT relative to the individual's balance ability [18]. Therefore, at this point, it is impossible to establish evidence-based guidelines for all BT modalities in healthy older adults (aged $\geq 65$ years). However, with reference to the best practice recommendations of Sherrington et al. [14], it is possible to present qualitative recommendations on training intensity during BT. Sherrington and colleagues propagate that if the goal is to improve balance and to prevent risk of falling in older adults, moderate to high challenging balance exercises should be conducted in a sufficient dose (at least $50 \mathrm{~h}$, this equate to around $2 \mathrm{~h}$ per week for 6 months). Furthermore, if the main aim is the prevention of falls in old age, practitioners should refer patients for the management of other risk factors where appropriate [14]. Falls have multiple interacting predisposing and precipitating causes [55]. Rubenstein [55] listed the important individual risk factor for falls according to 16 controlled studies and deduced the following order of priority: muscle weakness, balance deficit, gait deficit, visual deficit, mobility limitation, cognitive impairment, impaired functional status, and postural hypotension. Therefore, other intervention programs should be included in fall-preventive exercise program (e.g., strength or power training) to target a number of intrinsic fall-risk factors [55].

\subsection{Limitations}

A limitation of this systematic review is the poor methodological quality of the included studies. Only 6 out of 23 studies were classified as high quality according to the PEDro Scale (PEDro score $\geq 6$ ). In addition, many studies failed to report data necessary for computing SMD. Thus, future studies should report pre and post means and standard deviations for the investigated balance parameters. Moreover, further research of high methodological quality is needed to determine dose-response relationships of BT for specific training modalities such as training volume (i.e., number of exercises per training session, number of sets and/or repetitions per exercise, duration of a single balance exercise) in healthy older adults and to develop a feasible and effective method to regulate training intensity during BT. In addition, given that it is difficult to separate the impact of each training modality from that of others, that the heterogeneity between studies was considerable (i.e., $I^{2}=76-92 \%$ ) and that we were not able to take the grade of instability/training intensity that has been trained into account, the present findings are preliminary and have to be interpreted with caution. Further, the highlighted comparisons of dose-response relationships in old vs. young adults are limited because we indirectly 
compared age-specific dose-response relationships across studies using $\mathrm{SMD}_{\mathrm{bs}}$ and not in a single controlled study. Finally, findings from this meta-analysis do not allow conclusions with regard to fall-prevention. In other words, our detailed analyses revealed effective BT modalities to improve overall balance performance as well as more specific measures of static steady-state balance. It is unclear how these performance enhancements translate into reduced fall rates.

\section{Conclusions}

Unlike for endurance and resistance training, there are currently no evidence-based recommendations for effective BT protocols (i.e., optimal training modalities) in healthy older adults (aged $\geq 65$ years) available. Therefore, it is not surprising that the identified BT studies in older adults were heterogeneous with regard to the respective training modalities. To provide practitioners and therapists with evidence-based guidelines on effective BT protocols, we investigated the dose-response relationships of BT in healthy older adults. Our analyses revealed that a number of BT modalities (i.e., training period, training frequency, training volume) contribute to the improvements in measures of static/dynamic steady-state, proactive, and reactive balance as well as in the performance of balance test batteries in healthy older adults. An effective BT protocol for healthy older adults is characterized by a training period of 11-12 weeks, a training frequency of three sessions per week, a total number of 36-40 training sessions, a duration of 31-45 min of a single training session, and a total duration of 91-120 min of BT per week. When comparing our findings with those that were recently published in young healthy adults, it seems plausible to argue that almost the same BT protocols are effective in healthy young and older adults, in other words there appears to be no age effect. Given that only a few studies reported detailed information on the number of exercises per training session, the sets and/or repetitions per exercise, and the duration of single exercises dose-response relationships could not be drawn for these parameters. Hence, further research is necessary to prove and specify preliminary dose-response relationships of BT in healthy older adults. In addition, it would be interesting to find out in future studies whether dose-response relationships are significantly different in BT as compared with resistance and endurance training in healthy older adults.

\section{Compliance with Ethical Standards}

This work was supported by a grant from the German Research Foundation (MU 3327/2-1). Melanie Lesinski, Tibor Hortobágyi, Thomas Muehlbauer, Albert Gollhofer, and Urs Granacher declare that they have no conflict of interest.
Open Access This article is distributed under the terms of the Creative Commons Attribution 4.0 International License (http:// creativecommons.org/licenses/by/4.0/), which permits unrestricted use, distribution, and reproduction in any medium, provided you give appropriate credit to the original author(s) and the source, provide a link to the Creative Commons license, and indicate if changes were made.

\section{References}

1. Maki BE, Mcllroy WE. Postural control in the older adult. Clin Geriatr Med. 1996;12(4):635-58.

2. Era P, Sainio P, Koskinen S, et al. Postural balance in a random sample of 7979 subjects aged 30 years and over. Gerontology. 2006;52(4):204-13.

3. Hytonen M, Pyykko I, Aalto H, et al. Postural control and age. Acta Otolaryngol. 1993;113(2):119-22.

4. Abrahamova D, Hlavacka F. Age-related changes of human balance during quiet stance. Physiol Res. 2008;57(6):957-64.

5. Agrawal Y, Carey JP, Hoffman HJ, et al. The modified romberg balance test: normative data in US adults. Otol Neurotol. 2011;32(8):1309-11.

6. Abellan van Kan G, Rolland Y, Andrieu S, et al. Gait speed at usual pace as a predictor of adverse outcomes in community-dwelling older people an International Academy on Nutrition and Aging (IANA) Task Force. J Nutr Health Aging. 2009;13(10):881-9.

7. Shumway-Cook A, Brauer S, Woollacott M. Predicting the probability for falls in community-dwelling older adults using the timed up and go test. Phys Ther. 2000;80(9):896-903.

8. World Health Organisation. WHO global report on falls prevention in older age. Geneva: World Health Organisation; 2012.

9. Rubenstein LZ, Josephson KR. The epidemiology of falls and syncope. Clin Geriatr Med. 2002;18(2):141-58.

10. Howe TE, Rochester L, Neil F, et al. Exercise for improving balance in older people. Cochrane Database Syst Rev. 2011;11:CD004963.

11. Sherrington C, Whitney JC, Lord SR, et al. Effective exercise for the prevention of falls: a systematic review and meta-analysis. J Am Geriatr Soc. 2008;56(12):2234-43.

12. Hrysomallis C. Balance ability and athletic performance. Sports Med. 2011;41(3):221-32.

13. Granacher U, Muehlbauer T, Gruber M. A qualitative review of balance and strength performance in healthy older adults: impact for testing and training. J Aging Res. 2012;2012:708905.

14. Sherrington C, Tiedemann A, Fairhall N, et al. Exercise to prevent falls in older adults: an updated meta-analysis and best practice recommendations. N S W Public Health Bull. 2011;22(3-4):78-83.

15. Agmon M, Belza B, Nguyen HQ, et al. A systematic review of interventions conducted in clinical or community settings to improve dual-task postural control in older adults. Clin IntervAging. 2014;9:477-92.

16. Agency for Health Care Policy and Research. Clinical practice guideline. Rockville: AHCPR; 1992.

17. Lesinski M, Hortobagyi T, Muehlbauer T, et al. Dose-response relationships of balance training in healthy young adults: a systematic review and meta-analysis. Sports Med. 2015;45(4):557-76.

18. Farlie MK, Robins L, Keating JL, et al. Intensity of challenge to the balance system is not reported in the prescription of balance exercises in randomised trials: a systematic review. J Physiother. 2013;59(4):227-35.

19. Ackland TR, Elliott BC, Bloomfield J. Applied anatomy and biomechanics in sport ebook. 2nd ed. Champaign: Human Kinetics; 2009. 
20. Pew RW, Van Hemel SB. Technology for adaptive aging. Washington, DC: The National Academic Press; 2004.

21. Taube W, Gruber M, Gollhofer A. Spinal and supraspinal adaptations associated with balance training and their functional relevance. Acta Physiol. 2008;193(2):101-16.

22. Rand D, Miller WC, Yiu J, et al. Interventions for addressing low balance confidence in older adults: a systematic review and metaanalysis. Age Ageing. 2011;40(3):297-306.

23. Rubenstein LZ, Josephson KR. Falls and their prevention in elderly people: what does the evidence show? Med Clin North Am. 2006;90(5):807-24.

24. Shumway-Cook A, Woollacott MH. Motor control: translating research into clinical practice. 4th ed. Philadelphia: Lippincott; 2007.

25. Granacher U, Muehlbauer T, Zahner L, et al. Comparison of traditional and recent approaches in the promotion of balance and strength in older adults. Sports Med. 2011;41(5):377-400.

26. Muehlbauer T, Besemer C, Wehrle A, et al. Relationship between strength, power and balance performance in seniors. Gerontology. 2012;58(6):504-12.

27. Granacher U, Bridenbaugh SA, Muehlbauer T, et al. Age-related effects on postural control under multi-task conditions. Gerontology. 2011;57(3):247-55.

28. Maher CG, Sherrington C, Herbert RD, et al. Reliability of the PEDro scale for rating quality of randomized controlled trials. Phys Ther. 2003;83(8):713-21.

29. Deeks JJ, Higgins JPT. Statistical algorithms in Review Manager 5. In: Collaboration SMGoTC, editor; 2010. p. 1-11.

30. Hedges LV, Olkin I. Statistical methods for meta-analysis. Orlando: Academic Press; 1985.

31. Cohen J. Statistical power for the behavioral sciences. Hillsdale: Erlbaum; 1988.

32. Beling J, Roller M. Multifactorial intervention with balance training as a core component among fall-prone older adults. J Geriatr Phys Ther. 2009;32(3):125-33.

33. Gusi N, Carmelo Adsuar J, Corzo $\mathrm{H}$, et al. Balance training reduces fear of falling and improves dynamic balance and isometric strength in institutionalised older people: a randomised trial. J Physiother. 2012;58(2):97-104.

34. Kronhed ACG, Moller C, Olsson B, et al. The effect of short-term balance training on community-dwelling older adults. J Aging Phys Act. 2001;9(1):19-31.

35. Maughan KK, Lowry KA, Franke WD, et al. The dose-response relationship of balance training in physically active older adults. J Aging Phys Act. 2012;20(4):442-55.

36. Melzer I, Oddsson L. Improving balance control and self-reported lower extremity function in community-dwelling older adults: a randomized control trial. Clin Rehabil. 2013;27(3):195-206.

37. Nagai K, Yamada M, Tanaka B, et al. Effects of balance training on muscle coactivation during postural control in older adults: a randomized controlled trial. J GerontolA Biol Sci Med Sci. 2012;67(8):882-9.

38. Pfeifer K, Ruhleder M, Brettmann K, et al. Effekte eines koordinationsbetonten Bewegungsprogramms zur Aufrechterhaltung der Alltagsmotorik im Alter. Deut Z Sportmed. 2001;52(4):129-35.

39. Piao YJ, Kim K, Yu M, et al. Analysis on training effects of postural control for elderly adults. Int $\mathrm{J}$ Precis Eng Man. 2009;10(3):133-9.

40. Weerdesteyn V, Rijken H, Geurts AC, et al. A five-week exercise program can reduce falls and improve obstacle avoidance in the elderly. Gerontology. 2006;52(3):131-41.

41. Wolf SL, Barnhart HX, Ellison GL, et al. The effect of tai chi quan and computerized balance training on postural stability in older subjects. Atlanta FICSIT Group. Frailty and injuries: Cooperative studies on intervention techniques. Phys Ther. 1997;77(4):371-81.
42. Wolfson L, Whipple R, Derby C, et al. Balance and strength training in older adults: intervention gains and tai chi maintenance. J Am Geriatr Soc. 1996;44(5):498-506.

43. Yu CH, Piao YJ, Kim K, et al. The effects of an 8-weeks training on postural control for the elderly. Int J Precis Eng Man. 2014;15(1):161-8.

44. Granacher U, Gruber M, Gollhofer A. The impact of sensorimotor training on postural control in elderly men. Deut $\mathrm{Z}$ Sportmed. 2009;60(12):387-93.

45. Granacher U, Muehlbauer T, Bridenbaugh S, et al. Balance training and multi-task performance in seniors. Int $\mathrm{J}$ Sports Med. 2010;31(5):353-8

46. Judge JO, Whipple RH, Wolfson LI. Effects of resistive and balance exercises on isokinetic strength in older persons. J Am Geriatr Soc. 1994;42(9):937-46.

47. Jacobson BH, Thompson B, Wallace T, et al. Independent static balance training contributes to increased stability and functional capacity in community-dwelling elderly people: a randomized controlled trial. Clin Rehabil. 2011;25(6):549-56.

48. Leiros-Rodriguez R, Garcia-Soidan JL. Balance training in elderly women using public parks. J Women Aging. 2014;26(3):207-18.

49. Thiamwong L, Suwanno J. Effects of simple balance training on balance performance and fear of falling in rural older adults. Intern Gerontol. 2014;8:143-6.

50. Arampatzis A, Peper A, Bierbaum S. Exercise of mechanisms for dynamic stability control increases stability performance in the elderly. J Biomech. 2011;44(1):52-8.

51. Bierbaum S, Peper A, Arampatzis A. Exercise of mechanisms of dynamic stability improves the stability state after an unexpected gait perturbation in elderly. Age. 2013;35(5):1905-15.

52. Rossi LP, Pereira R, Brandalize M, et al. The effects of a perturbation-based balance training on the reactive neuromuscular control in community-dwelling older women: a randomized controlled trial. Hum Mov. 2013;14(3):238-46.

53. Weerdesteyn V, Nienhuis B, Duysens J. Exercise training can improve spatial characteristics of time-critical obstacle avoidance in elderly people. Hum Mov Sci. 2008;27(5):738-48.

54. Franco JR, Jacobs K, Inzerillo C, et al. Thse effect of the nintendo wii fit and exercise in improving balance and quality of life in community dwelling elders. Technol Health Care. 2012;20(2):95-115.

55. Rubenstein LZ. Falls in older people: epidemiology, risk factors and strategies for prevention. Age Ageing. 2006;35(2):ii37-41.

56. Gillespie LD, Robertson MC, Gillespie WJ, et al. Interventions for preventing falls in older people living in the community. Cochrane Database Syst Rev. 2012;9:CD007146.

57. Robertson MC, Campbell AJ, Gardner MM, et al. Preventing injuries in older people by preventing falls: a meta-analysis of individual-level data. J Am Geriatr Soc. 2002;50(5):905-11.

58. Chodzko-Zajko WJ, Proctor DN, Fiatarone Singh MA, et al. American College of Sports Medicine position stand. Exercise and physical activity for older adults. Med Sci Sports Exerc. 2009;41(7):1510-30.

59. Tiedemann A, Sherrington C, Close JC, et al. Exercise and sports science australia position statement on exercise and falls prevention in older people. J Sci Med Sport. 2011;14(6):489-95.

60. Fritz S, Lusardi M. White paper: "walking speed: the sixth vital sign". J Geriatr Phys Ther. 2009;32(2):46-9.

61. Perera S, Mody SH, Woodman RC, et al. Meaningful change and responsiveness in common physical performance measures in older adults. J Am Geriatr Soc. 2006;54(5):743-9.

62. Toulotte C, Thevenon A, Fabre C. Effects of training and detraining on the static and dynamic balance in elderly fallers and non-fallers: a pilot study. Disabil Rehabil. 2006;28(2):125-33. 